\title{
Heterotopic Pregnancy with an Intrauterine Twin Gestation and Ruptured Tubal Pregnancy: An Unusual Presentation
}

\author{
Sipra Bagchi ${ }^{1}$ Abhinav Aggarwal ${ }^{2,5}$ - Ashish Kumar Gupta ${ }^{3}$. \\ Aakriti Kapoor Aggarwal $^{4} \cdot$ Primal Sachdeva $^{3} \cdot$ Abhinav Pandit ${ }^{3}$
}

Received: 18 August 2015/Accepted: 11 November 2015/Published online: 30 November 2015

(C) Society of Fetal Medicine 2015

\begin{abstract}
Heterotopic pregnancy is diagnosed as coexistent presence of fetuses at two or more implantation sites. Its incidence is very rare with presence of live intrauterine twins being even rarer. Diagnosis in such cases is difficult with ultrasound being the investigation of choice. Management is primarily surgical. It is a clinical entity which is becoming even more common with widespread use of assisted reproductive techniques. A 20-year-old primigravida with 10 weeks of gestation presented with sudden onset pain on right side of abdomen. She had history of treatment for infertility for 1 year. Ultrasound revealed live intrauterine twin pregnancy with a heterogeneous lesion in right adnexal region and peritoneal free fluid. In concordance with clinical findings a diagnosis of heterotopic pregnancy with ruptured ectopic was made. Patient was managed through emergency laparotomy without disturbing the intrauterine twin gestation. Higher incidence of heterotopic pregnancy has been observed in patients undergoing assisted reproductive techniques and therefore warrant regular and detailed examination and follow up to avoid complications like ruptured ectopic pregnancy.
\end{abstract}

Abhinav Aggarwal

abhinavagg@gmail.com

1 Department of Gynaecology and Obstetrics, SRMSIMS, Bareilly, UP, India

2 A-1/237, First floor, Janak puri, New Delhi 110058, India

3 Department of Radiodiagnosis, SRMSIMS, Bareilly, UP, India

4 Medanta Hospital, Gurgaon, Haryana, India

5 City X Ray and Scan Clinic, Tilak Nagar, New Delhi, India
Keywords Assisted reproductive techniques . Heterotopic pregnancy $\cdot$ Live intrauterine twins . Ultrasound

\section{Introduction}

Heterotopic pregnancy (HP) is characterized by the presence of coexistent gestations at two or more implantation sites. It is a very rare medical condition with an incidence of around 1:30,000 in spontaneous natural conceptions [1]. The incidence is greater in pregnancies resulting from assisted reproduction techniques, varying from $1 / 100$ to $1 / 500$ and nearly as high as $1 \%$ in some series [2, 3]. Heterotopic triplets are even more uncommon and only few cases with tubal ectopic and coexisting twin intrauterine pregnancy are reported in literature. This medical condition can be hazardous to the intrauterine pregnancy and mother's life as well [1]. We present a rare case of heterotopic pregnancy following fertility treatment with intrauterine twin and ruptured right-sided tubal pregnancy, which was successfully surgically treated with preservation of the intrauterine pregnancy.

\section{Report of Case}

A 20-year-old primigravida with 10 weeks of pregnancy, presented to the emergency department with complaints of sudden onset of right-sided lower abdominal pain. She also complained of vomiting and spotting per vaginum. Selfurine pregnancy test was positive 15 days earlier. She gave history of infertility for one year for which she took fertility treatment, but records were not available. On physical examination she was pale, her blood pressure was 
100/70 $\mathrm{mm} \mathrm{Hg}$ and heart rate was 120 beats/min. Abdominal examination demonstrated fullness in lower abdomen with diffuse abdominal tenderness that was maximal in the right iliac fossa and signs of peritonism. Per speculum examination demonstrated a closed posterior cervical os with minimal bleeding. Cervical tenderness was elicited during bimanual examination, with cervical excitation maximal in the right adnexa. Uterus was around 12-14 weeks size. Her hemoglobin was $6.8 \mathrm{~g} / \mathrm{dL}$. Ultrasonography demonstrated two intrauterine gestational sacs with fetuses (Fig. 1). Cardiac activity was present in both the fetuses (Fig. 2). The crown rump lengths (CRL) of both the fetuses were $30 \mathrm{~mm}$ corresponding to gestation of 9 weeks and 6 days. The right adnexa showed a heterogenous mass of approximately $10.0 \mathrm{~cm} \times 4.0 \mathrm{~cm}$ (Fig. 3). Moderate amount of free fluid with internal echoes was present in the pelvis.

A provisional diagnosis of a heterotopic triplet pregnancy with live intrauterine twin gestation and ruptured right ectopic gestation was made. The patient underwent

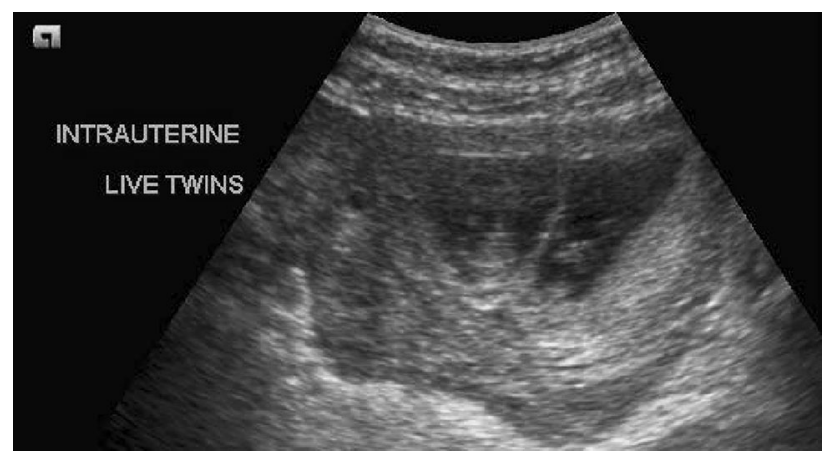

Fig. 1 Transabdominal ultrasound showing live intrauterine twins

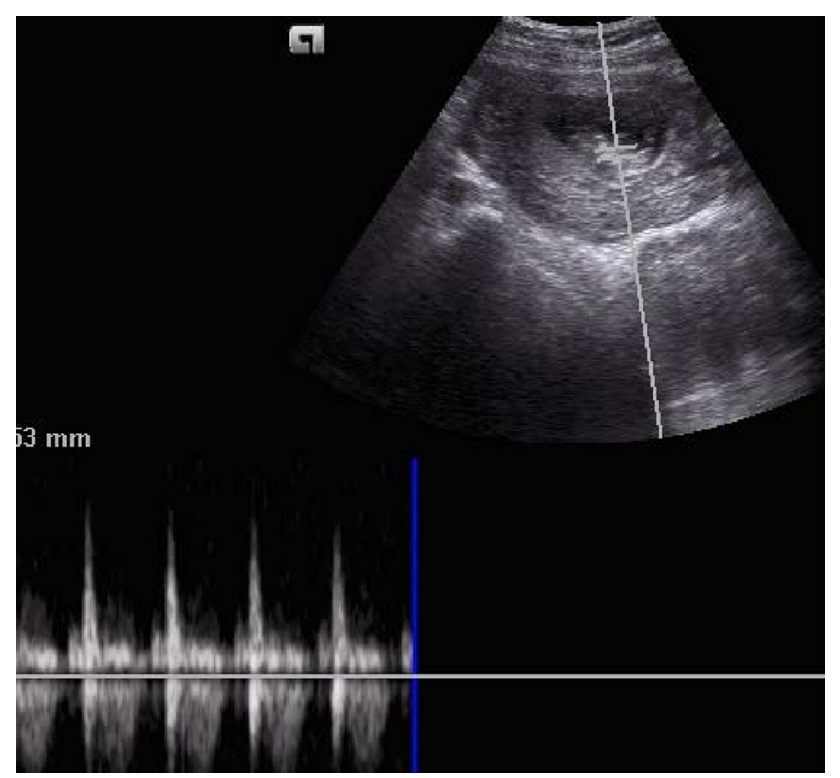

Fig. 2 Transabdominal ultrasound showing fetal cardiac activity

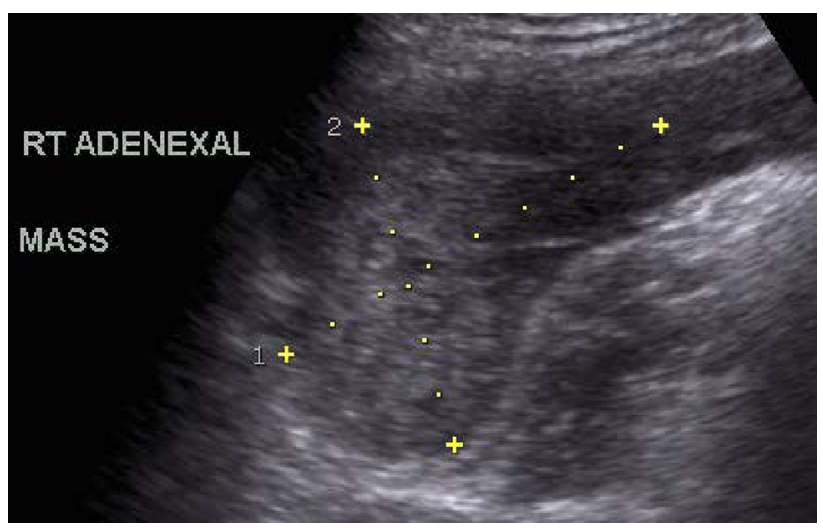

Fig. 3 Transabdominal ultrasound showing heterogeneous right adnexal lesion

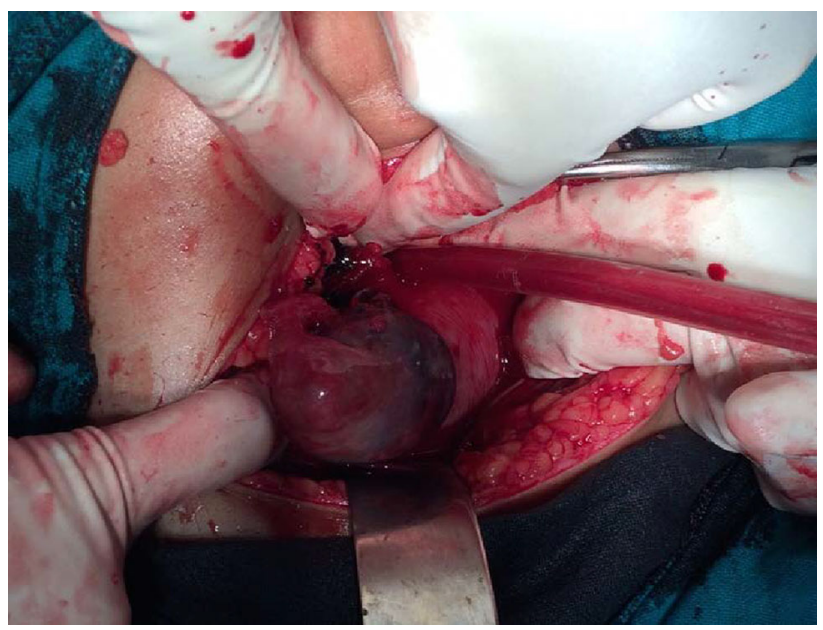

Fig. 4 Intraoperative photograph showing right sided tubal pregnancy with drainage tube in situ

emergency laparotomy. There was ruptured right-sided tubal pregnancy with hemoperitoneum (Fig. 4). Uterus was around 12 weeks size. Right-sided salpingectomy was done. All precautions were taken to not disturb the intrauterine gestation. Two units of blood were transfused postoperatively. Postoperative recovery was uneventful and patient was discharged on day 8 after stitch removal. She is under regular follow up and is currently in 28th week of gestation with no complications.

\section{Discussion}

HP is usually diagnosed as one or more intrauterine pregnancies co-existing with an ectopic pregnancy, which can be tubal, ovarian, cervical, cornual or abdominal. Of these, tubal pregnancies are the most common [4]. The higher incidence of HP in assisted reproductive pregnancies as compared to natural spontaneous conception is due to 
transfer of embryos by ART techniques into affected tubes and peristaltic movements do not expel these embryos. Previous history of tubal surgery or pelvic inflammatory disease also predispose to HP [5].

Abdominal pain, adnexal mass, peritoneal irritation and an enlarged uterus are signs and symptoms [6]. Ultrasonography especially through transvaginal approach provides an important aid in diagnosis. The presence of cardiac activity in both intrauterine and ectopic fetuses confirms the diagnosis of HP [4]. Culdocentesis has proven to be beneficial in the diagnosis of ruptured ectopic pregnancies. Serial $\beta$-HCG levels are not very useful in the diagnosis of HP since subnormal levels of hormone production by ectopic pregnancy are masked by higher placental production from the intrauterine pregnancy [5]. Although most of the reported HPs have singleton intrauterine pregnancies, triplet and quadruplet HP have been rarely reported [7]. However their incidence has been increasing in the last decade due to increased use of assisted reproduction techniques. There is a case report of triplet heterotopic pregnancy with bilateral tubal ectopics and single live intrauterine pregnancy [8].

Some of the relatively older studies have reported that the use of ultrasound is not very sensitive for the detection of HP $[5,8]$.

Ectopic pregnancies are treated by laparoscopy or laparotomy depending upon the clinical profile of the patient [9]. The main precaution to be taken is not to disturb the live intrauterine pregnancy. With advancements in surgical and anaesthetic techniques the success of surgical management has increased over the years.

\section{Conclusion}

Heterotrophic pregnancy is rare but potentially a lifethreatening condition. Every patient undergoing assisted reproductive techniques needs to be thoroughly and regularly investigated and followed up for procedure-associated complications like heterotopic pregnancy. A high index of suspicion is necessary for early and timely diagnosis to achieve optimal clinical outcomes.

\section{Compliance with Ethical Standards}

Conflict of interest None.

\section{References}

1. Felekis T, Akrivis C, Tsirkas P, Korkontzelos I. Heterotopic triplet pregnancy after in vitro fertilization with favorable outcome of the intrauterine twin pregnancy subsequent to surgical treatment of the tubal pregnancy. Case Rep Obstet Gynecol. 2014;2014:356131.

2. Varras M, Akrivis C, Hadjopoulos G, Antoniou N. Heterotopic pregnancy in a natural conception cycle presenting with tubal rupture: a case report and review of the literature. Eur J Obstet Reprod Biol. 2003;106:79-82.

3. Tal J, Haddad S, Gordon N, Timor-Tritsch I. Heterotopic pregnancy after ovulation induction and assisted reproductive techniques: a literature review from 1971 to 1993 . Fertil Steril. 1996;66:1-12.

4. Bright DA, Craupp FB. Heterotopic pregnancy: a re-evaluation. J Am Board Fam Pract. 1990;3:125-8.

5. Tandon R, Goel P, Saha PK, Devi L. Spontaneous heterotopic pregnancy with tubal rupture: a case report and review of the literature. J Med Case Rep. 2009;3:8153.

6. Reece EA, Petrie RH, Sirmans MF, Finster M, Todd WD. Combined intrauterine and extrauterine gestations: a review. Am J Obstet Gynecol. 1983;146:323-30.

7. Sherer DM, Scibetta JJ, Sanko SR. Heterotopic quadruplet gestation with laparoscopic resection of ruptured interstitial pregnancy and subsequent successful outcome of triplets. Am J Obstet Gynecol. 1995;172:216.

8. Pan HS, Chuang J, Chiu SF, Hsieh BC, Lin YH, Tsai YL, et al. Heterotopic triplet pregnancy: report of a case with bilateral tubal pregnancy and an intrauterine pregnancy: case report. Hum Reprod. 2002;17(5):1363-6.

9. Arsala L, Danso D. spontaneous heterotopic triplet pregnancy with tubal rupture: a case report and literature review. J Invest Med. 2014;2:2. 